\title{
UNIQUE CONTINUATION AND DECAY FOR THE KORTEWEG-DE VRIES EQUATION WITH LOCALIZED DAMPING
}

\author{
Ademir FERnANDo PAZOTO ${ }^{1}$
}

\begin{abstract}
This work is devoted to prove the exponential decay for the energy of solutions of the Korteweg-de Vries equation in a bounded interval with a localized damping term. Following the method in Menzala (2002) which combines energy estimates, multipliers and compactness arguments the problem is reduced to prove the unique continuation of weak solutions. In Menzala (2002) the case where solutions vanish on a neighborhood of both extremes of the bounded interval where equation holds was solved combining the smoothing results by T. Kato (1983) and earlier results on unique continuation of smooth solutions by J.C. Saut and B. Scheurer (1987). In this article we address the general case and prove the unique continuation property in two steps. We first prove, using multiplier techniques, that solutions vanishing on any subinterval are necessarily smooth. We then apply the existing results on unique continuation of smooth solutions.
\end{abstract}

Mathematics Subject Classification. 35B40, 35Q53.

Received October 14, 2003. Revised October 19, 2004.

\section{INTRODUCTION}

This paper is devoted to study the Unique Continuation Property (UCP) and the exponential decay of the solutions of the Korteweg-de Vries (KdV) equation in a bounded interval under the presence of a localized damping:

$$
\left\{\begin{array}{l}
u_{t}+u_{x}+u_{x x x}+u u_{x}+a(x) u=0 \quad \text { in }(0, L) \times(0, T), \\
u(0, t)=u(L, t)=0, \quad t \in(0, T), \\
u_{x}(L, t)=0, \quad t \in(0, T) \\
u(0)=u_{0}, \quad \text { on }(0, L) .
\end{array}\right.
$$

All along the paper we assume that the function $a=a(x)$ satisfies

$$
\left\{\begin{array}{l}
a \in L^{\infty}(0, L) \text { and } a(x) \geq a_{0}>0 \text { a.e. in } \omega, \\
\text { where } \omega \text { is a nonempty open subset of }(0, L) .
\end{array}\right.
$$

Keywords and phrases. Unique continuation, decay, stabilization, KdV equation, localized damping.

${ }^{1}$ Instituto de Matemática, Universidade Federal do Rio de Janeiro, PO Box 68530, CEP 21945-970, Rio de Janeiro, RJ, Brasil; ademir@acd.ufrj.br

(c) EDP Sciences, SMAI 2005 
Note that multiplying the equation by $u$ and integrating in $(0, L)$ one gets

$$
\frac{\mathrm{d} E}{\mathrm{~d} t}=-\int_{0}^{L} a(x)|u(x, t)|^{2} \mathrm{~d} x-\frac{1}{2}\left|u_{x}(0, t)\right|^{2}
$$

with

$$
E(t)=\frac{1}{2} \int_{0}^{L}|u(x, t)|^{2} \mathrm{~d} x .
$$

This indicates that the term $a(x) u$ in the equation plays the role of a feedback damping mechanism. Consequently, $E(t)$ is a nonincreasing function and a rate of decay of solutions is expected.

In the absence of the internal damping mechanism, i.e., when $a \equiv 0$, the equation in (1) was first derived in $[9]$ as a model for propagation of surface water waves along a channel. Its original form was

$$
\eta_{t}=\frac{3}{2} \sqrt{\frac{g}{l}}\left(\frac{1}{2} \eta^{2}+\frac{2}{3} \alpha \eta+\frac{1}{3} \sigma \eta_{x x}\right)_{x}
$$

where $\eta$ is the surface elevation above the equilibrium level $l, \alpha$ are small constants related to the uniform motion of the liquid, $g$ is the gravitational constant, and $\sigma=l^{3} / 3-T l / \rho g$ with surface capillary tension $T$ and density $\rho$. It is the first model for which explicit solitons were found to exist.

At present it is known that equation (5) is not only a good model for water waves but also a very useful approximation model in nonlinear studies whenever one wishes to include and balance weak nonlinear and dispersive effects. In particular, the equation is commonly accepted as a mathematical model for the unidirectional propagation of small-amplitude long waves in nonlinear dispersive systems. In such applications, the unknown is typically an amplitude or velocity, $x$ is often proportional to distance in the direction of propagation, and $t$ is proportional to the elapsed time.

For the boundary value problem (1) under consideration, according to the above dissipation law (3), even when $a=0$, the energy is dissipated through the extreme $x=0$. However, as far as we know, there is no available result on the decay of solutions of (1) on the case $a \equiv 0$. On the other hand, when $a \equiv 1$, it is straightforward to see from (3) that the energy decays uniformly exponentially as $t \rightarrow \infty$. The same holds when $a(x) \geq a_{0}>0$ a.e. in $[0, L]$. The problem of stabilization when the damping is effective only on a bounded subset of the interval $(0, L)$ is much more subtle and, in view of $(3)$, the problem of the exponential decay of $E(t)$ can be stated in the following equivalent form: To find $T>0$ and $C>0$ such that

$$
E(0) \leq C \int_{0}^{T}\left[\int_{0}^{L} a(x) u^{2}(x, t) \mathrm{d} x+u_{x}^{2}(0, t)\right] \mathrm{d} t
$$

holds for every finite energy solution of (1). Indeed, from (6) and (3), we have that $E(T) \leq \gamma E(0)$ with $0<\gamma<1$, which combined with the semigroup property, allow us to derive the exponential decay of $E(t)$.

This paper is devoted to analyze this problem.

In [12], the case where the damping term $a(x) u$ is active simultaneously in a neighborhood of both extremes of the interval $(0, L)$ was addressed. It was proved that for all $T>0$ and any solution of (1) with initial data satisfying $E(0) \leq R$ inequality (6) holds with a constant $C=C(R, T)$. As a consequence of this it was shown that, for any $R>0$, there exist positive constants $C=C(R)$ and $\alpha(R)$ satisfying

$$
E(t) \leq C(R) E(0) \mathrm{e}^{-\alpha(R) t}, \quad \forall t>0
$$

provided $E(0) \leq R$. The proof in [12] follows closely the multiplier techniques developed in [15] for the analysis of controllability properties. However, when using multipliers, the nonlinearity produces extra terms that, in [12], were handled by compactness. 
In fact, proceeding as in [12] the problem of obtaining (6) is reduced to showing that the unique solution of (1), such that, $a(x) u=0$ everywhere and $u_{x}(0, t)=0$ for all time $t$, has to be the trivial one.

This problem may be viewed as a unique continuation one since $a u=0$ implies that $u=0$ in $\{a>0\} \times(0, T)$. However, the existing unique continuation results (see [17]) do not apply directly since the solutions we are dealing with are weak, with initial data in $L^{2}(0, L)$ and the regularity conditions required to derive Calerman inequalities are not fulfilled. We point out that such inequalities are those reminiscent of the classical Carleman estimates in which the lower order terms (with bounded coefficients or even with unbounded coefficients under suitable integrability conditions) of the equation can be controlled in some weighted norms by the principal part of the operator (see for instance $[5,6,19,24]$ ).

When $\omega$ contains a neighborhood of both extremes $x=0, L$, the problem was solved in [12] in two steps. First, extending the solution by zero outside the interval $(0, L)$, we get a compactly supported (in space) solution of the Cauchy problem for the KdV equation on the whole line. We then apply the classical smoothing properties as in $[8,10]$, showing that the solution is smooth. This allows applying the unique continuation results in [20] on smooth solutions to conclude that $u \equiv 0$.

When $\omega$ does not have the property above, i.e., when $\omega$ does not contain a neighborhood of both extremes $x=0, L$, this argument cannot be applied. Consequently, one has to improve the regularity of the solution working within the bounded interval $(0, L)$. A possible way of attacking this problem would be to develop Carleman inequalities for weak solutions of $\mathrm{KdV}$ equations as it has been done for the wave equation in [16] and for the heat equation in [7]. This would allow proving directly the unique continuation of weak solutions without going through the intermediate step of proving its regularity. We have not pursued this approach since the argument we shall develop here of proving the regularity of the solutions that vanish on a subinterval is quite natural. It was used before for linear, time invariant wave and plate equations by Rauch and Taylor [14] and Zuazua [22]. More recently the problem of decay for locally dissipated semilinear wave equation was analyzed by Dehman, Lebeau and Zuazua in [3]. There the authors developed an argument based on the propagation of singularities and Strichartz's inequalities to prove that solutions vanishing on the exterior of a ball are smooth, which allowed them to apply the existing results on unique continuation.

Consequently, this article may be considered as a new contribution in the subject of proving unique continuation properties of weak solutions of partial differential equations, this time in the context of KdV equations.

As far as we know, the situation we are considering here has not been addressed in the literature yet since, to our knowledge, the existing results on unique continuation for $\mathrm{KdV}$ like equations (see [17,20]) require the solution $u$ to be in $L^{\infty}\left(0, T ; H^{s}(0, L)\right)$ with $s>3 / 2$.

Let us now describe our strategy of proof in some more detail. We first differentiate the equation in (1) with respect to $t$ and analyze the regularity of $v=u_{t}$, which is a solution of

$$
\left\{\begin{array}{l}
v_{t}+v_{x}+v_{x x x}+(u(x, t) v)_{x}+a(x) v=0 \quad \text { in }(0, L) \times(0, T), \\
v(0, t)=v(L, t)=0, \quad t \in(0, T) \\
v_{x}(L, t)=0, \quad t \in(0, T) \\
v(0)=v_{0}, \quad \text { on }(0, L)
\end{array}\right.
$$

where $u \in L^{2}\left(0, T ; H_{0}^{1}(0, L)\right) \cap L^{\infty}\left(0, T ; L^{2}(0, L)\right)$ is the weak solution of $(1)$ and $v_{0}=v(x, 0)=u_{t}(x, 0)$ in $H^{-3}(0, L)$. Of course, since $u \equiv 0$ in $\omega \times(0, T), v \equiv 0$ on $\omega \times(0, T)$ as well, $\omega$ being the subinterval where the damping potential $a$ is effective.

Observe that the above model (8) can be viewed as a linearized KdV equation. Therefore, inspired by the work of Rosier [15,21], we argue as in the linear case, combining multiplier techniques and the so called "compactnessuniqueness" argument (see [22]), which is useful to handle the extra terms that the "potential" $u(x, t)$ produces in the inequality. This allows us proving the fact that $v \equiv 0$ in $\omega \times(0, T)$ implies the extra regularity property $v \in L^{2}\left(0, T ; H_{0}^{1}(0, L)\right) \cap L^{\infty}\left(0, T ; L^{2}(0, L)\right)$ which yields enough regularity on $u$ to apply the unique continuation results obtained in [17] by means of Carleman inequalities. 
Note that the regularity result we prove on $v$ exhibits a definite gain of regularity since, in principle, the initial datum of $v$ belongs to $H^{-3}(0, L)$. The gain of regularity comes from the fact that $v \equiv 0$ on $\omega \times(0, T)$. Of course, we end up showing that $v \equiv 0$ everywhere, but this intermediate step on the gain of regularity plays a central role in the argument.

In the next section we shall address all arguments in detail, but first, for the sake of completeness, we give a sketch of the proof of the existence and uniqueness of global solutions of (8).

Before closing this section we note that the exponential decay rate (7) we get for solutions of (1) is uniform for initial data in balls $B_{R}$ of $L^{2}(0, L)$. But the results obtained in this paper do not provide any estimate on how the decay rate depends on the radius $R$ of the ball. This has been done for nonlinear models, as far as we know, in very few cases and always using some structural conditions on the nonlinearity. We refer to [23] for the case of the semilinear wave equation with localized damping in which the uniform exponential decay is proved and to [13] for the analysis of the von Kàrmàn system of thermoelastic plates where an explicit estimate on how the decay rate tends to zero as $R \rightarrow \infty$ is provided.

The problem of exponential decay we address here is closely related with the problem of controllability. Rosier in [15] (see also [2]) proved that the underlying linear equation without damping is exactly controllable by means of a single boundary control except when the length of the space interval $L$ lies in a countable set of critical lengths, of the form

$$
\mathcal{E}=\left\{\frac{2 \pi}{\sqrt{3}} \sqrt{k^{2}+k l+l^{2}}, k \text { and } l \text { are positive natural numbers }\right\}
$$

This was done using multiplier techniques and Lions' HUM method (see [11]). As we mentioned before, our methods are strongly inspired in those introduced in [15]. The critical lengths in (9) are such that there are eigenfunctions of the linear problem for which the observability inequality (6) leading to controllability fails when $a \equiv 0$. In this context of the stabilization problem, they correspond to solutions that, when $a \equiv 0$, are undamped, i.e., such that $u_{x}(0, t)=0$ for all time $t$. By a linearization argument a local controllability result for the semilinear equation was also proved in [15]. Later on, Zhang in [21] proved that using three controls, acting on all the boundary conditions, controllability holds for all values of $L$. More recently Crépeau and Coron proved in [2] that, for some critical values of the length $L$, the nonlinear system is controllable. This suggests that the exponential decay of solutions of the nonlinear problem may hold even when $a \equiv 0$. But this is an open problem. Indeed, the problem of stabilization when $a \equiv 0$ is mainly open. When $L \notin \mathcal{E}$ the fact that the linear semigroup decays exponentially allows showing that small solutions of the nonlinear problem (1) decay as well, even if $a \equiv 0$. But nothing is known about the case $L \in \mathcal{E}$ or for large solutions when $L \notin \mathcal{E}$ and $a \equiv 0$.

Let us briefly describe the sections of this paper: In Section 2 we present the main results of this work. Section 3 is devoted to prove our main result, i.e., the UCP of weak solutions of (1). In Section 4 we present a close related result and in Section 5 we formulate an open problem.

\section{Statement of the main Results}

For the sake of completeness, we state the existence and uniqueness result for problem (1) obtained in [12]:

Theorem 2.1 (see [12], Sect. 3). For any $u_{0} \in L^{2}(0, L)$ problem (1) has a unique mild solution $u \in L^{2}\left(0, \infty ; H_{0}^{1}(0, L)\right) \cap L^{\infty}\left(0, \infty ; L^{2}(0, L)\right)$. Furthermore, according to (3.23) in [12], for any $T>0$,

$$
\|u\|_{L^{2}\left(0, T ; H_{0}^{1}(0, L)\right)}^{2} \leq \frac{(8 T+2 L)}{3}\left\|u_{0}\right\|_{L^{2}(0, L)}^{2}+\frac{T C}{27}\left\|u_{0}\right\|_{L^{2}(0, L)}^{4}
$$

where $C$ is a positive constant. 
The main results of this paper can be summarized as follows:

Theorem 2.2. Let $u$ be the solution of problem (1) obtained in Theorem 2.1 and $\omega$ and $a=a(x)$ as in (2). Let $0<T<\infty$. If

$$
u_{x}(0, t)=0 \text { and } u \equiv 0 \text { in } \omega \times(0, T)
$$

then,

$$
u \in L^{2}\left(0, T ; H^{3}(0, L)\right) \cap H^{1}\left(0, T ; L^{2}(0, L)\right) .
$$

Consequently, the UCP holds and, therefore, $u \equiv 0$.

Our main result on exponential decay is as follows:

Theorem 2.3. For any $L>0$, any damping potential a satisfying (2) and $R>0$, there exist $c=c(R)>0$ and $\mu=\mu(R)>0$ such that

$$
E(t) \leq c\left\|u_{0}\right\|_{L^{2}(0, L)}^{2} \mathrm{e}^{-\mu t}
$$

holds for all $t \geq 0$ and any solution of (1) with $u_{0} \in L^{2}(0, L)$ such that $\left\|u_{0}\right\|_{L^{2}(0, L)} \leq R$.

As mentioned in the introduction, once Theorem 2.2 is known, Theorem 2.3 holds immediately applying the methods in [12]. Indeed, to prove Theorem 2.3 it is sufficient to show that for any $T>0$ and $R>0$ there exists a constant $c=c(T, R)>0$ such that (6) holds for every solution with $\left\|u_{0}\right\| \leq R$. Once this is done, (6) together with the energy dissipation law (3) yields (10).

Now, in order to prove the claim (6), we multiply the equation in $(1)$ by $(T-t) u$ and integrate in $(0, L) \times(0, T)$. Then, according to (3.25), [12], we deduce that

$$
\left\|u_{0}\right\|_{L^{2}(0, L)}^{2} \leq \frac{1}{T} \int_{0}^{T} \int_{0}^{L}|u|^{2} \mathrm{~d} x \mathrm{~d} t+\int_{0}^{T}\left|u_{x}(0, t)\right|^{2} \mathrm{~d} t+2 \int_{0}^{T} \int_{0}^{L} a(x)|u|^{2} \mathrm{~d} x \mathrm{~d} t .
$$

Thus, in order to prove (6) it is sufficient to prove that

$$
\int_{0}^{T} \int_{0}^{L}|u|^{2} \mathrm{~d} x \mathrm{~d} t \leq C\left\{\int_{0}^{T}\left|u_{x}(0, t)\right|^{2} \mathrm{~d} t+2 \int_{0}^{T} \int_{0}^{L} a(x)|u|^{2} \mathrm{~d} x \mathrm{~d} t\right\}
$$

where $C=C(R, T)>0$.

It is precisely at this point that the UCP is crucially needed. In fact, to prove (11) we argue by contradiction and use the so-called "compacteness-uniqueness argument". This allows us to build a sequence of functions $\left\{u_{n}\right\} \in L^{\infty}\left(0, T ; L^{2}(0, L)\right) \cap L^{2}\left(0, T ; H_{0}^{1}(0, L)\right)$ that solve $(1)$, satisfying $\left\|u_{n}(., 0)\right\| \leq R$ and such that

$$
\lim _{n \rightarrow \infty} \frac{\left\|u_{n}\right\|_{L^{2}\left(0, T ; L^{2}(0, L)\right)}^{2}}{\int_{0}^{T}\left|u_{n, x}(0, t)\right|^{2} \mathrm{~d} t+\int_{0}^{T} \int_{0}^{L} a(x) u_{n}^{2} \mathrm{~d} x \mathrm{~d} t}=\infty .
$$

Let $\lambda_{n}=\left\|u_{n}\right\|_{L^{2}\left(0, T ; L^{2}(0, L)\right)}$ and define $w_{n}(x, t)=u_{n}(x, t) / \lambda_{n}$. For each $n \in \mathbb{N}$ the function $w_{n}$ solves

$$
\left\{\begin{array}{l}
w_{n, t}+w_{n, x}+w_{n, x x x}+\lambda_{n} w_{n} w_{n, x}+a(x) w_{n}=0 \quad \text { in }(0, L) \times(0, T), \\
w_{n}(0, t)=w_{n}(L, t)=w_{n, x}(L, t)=0, \quad t \in(0, T), \\
w_{n}(x, 0)=w_{n, 0}, \quad \text { in }(0, L)
\end{array}\right.
$$

and satisfy

$$
\left\|w_{n}\right\|_{L^{2}\left(0, T ; L^{2}(0, L)\right)}=1
$$


and

$$
\left\{\int_{0}^{T}\left|w_{n, x}(0, t)\right|^{2} \mathrm{~d} t+\int_{0}^{L} \int_{0}^{L} a(x) w_{n}^{2} \mathrm{~d} x \mathrm{~d} t\right\} \rightarrow 0 \text { as } n \rightarrow \infty .
$$

Proceeding as in Theorem 3.1, [12], we may prove that $w_{n} \rightarrow w$ strongly in $L^{2}\left(0, T ; L^{2}(0, L)\right)$, where $w$ is the unique solution of

satisfying the extra conditions

$$
\left\{\begin{array}{l}
w_{t}+w_{x}+w_{x x x}+w w_{x}+a(x) w=0 \quad \text { in }(0, L) \times(0, T), \\
w(0, t)=w(L, t)=0, \quad t \in(0, T) \\
w_{x}(L, t)=0, \quad t \in(0, T)
\end{array}\right.
$$

$$
\left\{\begin{array}{l}
w_{x}(0, t)=0, \quad t \in(0, T), \\
w \equiv 0, \quad \text { in } \omega \times(0, T) .
\end{array}\right.
$$

Thus, by the UCP given by Theorem 2.2 , we have $w \equiv 0$ in $(0, L) \times(0, T)$. This contradicts (12) and, necessarily, (11) has to be true. Indeed, since $w_{n} \rightarrow w$ strongly in $L^{2}\left(0, T ; L^{2}(0, L)\right)$, it follows from (12) that $\|w\|_{L^{2}\left(0, T ; L^{2}(0, L)\right)}=1$.

Remark. Note that Holmgren's Uniqueness Theorem may not be applied for (14)-(15), since we are dealing with a semilinear equation.

\section{Proof of Theorem 2.2}

In the sequel, we prove some technical results constituting the basic ingredients for obtaining the regularity result in Theorem 2.2 .

Lemma 3.1. Let $u$ be the solution of problem (1) obtained in Theorem 2.1. Then, problem (8) has a unique mild solution $v \in L^{2}\left(0, T ; H_{0}^{1}(0, L)\right) \cap L^{\infty}\left(0, T ; L^{2}(0, L)\right)$ whenever $v_{0} \in L^{2}(0, L)$.

Proof. Firstly, let us consider model (8) in the absence of the term $f=(u(x, t) v)_{x}$ :

$$
\left\{\begin{array}{l}
v_{t}+v_{x}+v_{x x x}+a(x) v=0 \quad \text { in }(0, L) \times(0, T), \\
v(0, t)=v(L, t)=0, \quad t \in(0, T) \\
v_{x}(L, t)=0, \quad t \in(0, T) \\
v(0)=v_{0}, \quad \text { on }(0, L)
\end{array}\right.
$$

It is well known (see Sect. 2, $[12,15])$ that the above model generates a semigroup of contractions $\{S(t)\}_{t \geq 0}$ in $L^{2}(0, L)$. Furthermore, if we multiply the equation in (16) by $v$ and $x v$, and integrate over $(0, L) \times(0, T)$ we obtain

and

$$
\|v(\cdot, T)\|_{L^{2}(0, L)}^{2}=\left\|v_{0}\right\|_{L^{2}(0, L)}^{2}-\int_{0}^{T}\left|v_{x}(0, t)\right|^{2} \mathrm{~d} t-2 \int_{0}^{T} \int_{0}^{L} a(x)|v|^{2} \mathrm{~d} x \mathrm{~d} t
$$

$$
\int_{0}^{T} \int_{0}^{L} v_{x}^{2} \mathrm{~d} x \mathrm{~d} t+\frac{1}{3} \int_{0}^{L} x v^{2}(x, T) \mathrm{d} x+\frac{2}{3} \int_{0}^{T} \int_{0}^{L} x a(x) v^{2} \mathrm{~d} x \mathrm{~d} t=\frac{1}{3} \int_{0}^{T} \int_{0}^{L} v^{2} \mathrm{~d} x \mathrm{~d} t+\frac{1}{3} \int_{0}^{L} x v_{0}^{2}(x) \mathrm{d} x
$$

respectively. 
Then, we may deduce that $S(\cdot)$ satisfies the following properties

$$
\begin{gathered}
\left\|S(t) v_{0}\right\|_{L^{2}(0, L)} \leq\left\|v_{0}\right\|_{L^{2}(0, L)}, \\
\left\|S(.) v_{0}\right\|_{L^{2}\left(0, T ; H_{0}^{1}(0, L)\right)} \leq c(L+T)\left\|v_{0}\right\|_{L^{2}(0, L)},
\end{gathered}
$$

for all $0 \leq t \leq T$ and $v_{0} \in L^{2}(0, L)$.

Using the variation of constants formula, system (8) may be written in the following integral form

$$
v(t)=S(t) v_{0}+\int_{0}^{t} S(t-s)[u(x, s) v]_{x} \mathrm{~d} s=\Phi[v](t) .
$$

Thus, the problem of existence and uniqueness for (8) is reduced to finding a fixed point of $\Phi$. To do that, we proceed as in Section 3 [12], and show that $\Phi$ is a contraction from a suitable ball $B_{R}$ of

$$
X_{T}=L^{2}\left(0, T ; H_{0}^{1}(0, L)\right) \cap L^{\infty}\left(0, T ; L^{2}(0, L)\right)
$$

into itself when $T>0$ is small enough (both $R$ and $T$ depend on the size of the initial data $v_{0}$ in $L^{2}(0, L)$ and of the potential $u=u(x, t)$ in $\left.L^{\infty}\left(0, T ; L^{2}(0, L)\right) \cap L^{2}\left(0, T ; H_{0}^{1}(0, L)\right)\right)$.

First, observe that $\Phi$ maps continuously $X_{T}$ into itself. Indeed, according to (17), $S(\cdot) u_{0}$ lies in $X_{T}$. On the other hand, the function

solves the system

$$
y(t)=\int_{0}^{t} S(t-s)[u(x, t) v]_{x} \mathrm{~d} s
$$

$$
\left\{\begin{array}{l}
y_{t}+y_{x}+y_{x x x}=(u(x, t) v)_{x}=f \quad \text { in }(0, L) \times(0, T), \\
y(0, t)=y(L, t)=0, \quad t \in(0, T) \\
y_{x}(L, t)=0, \quad t \in(0, T) \\
y(0)=0, \quad \text { on }(0, L)
\end{array}\right.
$$

Then, by Proposition 4.1 in [15] (see also the estimates below), it follows that $y \in X_{T}$ and the map $f \in$ $L^{1}\left(0, T ; L^{2}(0, L)\right) \rightarrow y \in X_{T}$ is continuous. Moreover, the map that to each $u \in L^{2}\left(0, T ; H_{0}^{1}(0, L)\right)$ associates $(u(x, t) v)_{x}=u_{x}(x, t) v+u(x, t) v_{x}=f \in L^{1}\left(0, T ; L^{2}(0, L)\right)$ is continuous as well. This shows that $\Phi$ maps continuously $X_{T}$ into itself.

Now let us prove that $\Phi$ is a contraction in a suitable ball of $X_{T}$ when $T>0$ is small enough:

$$
\begin{aligned}
\Phi(v)-\Phi(w) & =\int_{0}^{t} S(t-s)\left([u(x, s) v]_{x}-[u(x, s) w]_{x}\right) \mathrm{d} s \\
& =\int_{0}^{t} S(t-s)\left(u(x, s)\left(v_{x}-w_{x}\right)+u_{x}(x, s)(v-w)\right) \mathrm{d} s
\end{aligned}
$$

and according to the previous analysis,

$$
\|\Phi(v)-\Phi(w)\|_{X_{T}} \leq c(L+T)\left\|u(x, s)\left(v_{x}-w_{x}\right)+u_{x}(x, s)(v-w)\right\|_{L^{1}\left(0, T ; L^{2}(0, L)\right)} .
$$

Then, it follows from triangular inequality and Hölder's inequality that

$$
\begin{aligned}
\|\Phi(v)-\Phi(w)\|_{X_{T}} \leq c(L+T)\left\{\|u\|_{L^{2}\left(0, T ; L^{\infty}(0, L)\right)}\left\|v_{x}-w_{x}\right\|_{L^{2}\left(0, T ; L^{2}(0, L)\right)}\right. & \\
& \left.+\left\|u_{x}\right\|_{L^{2}\left(0, T ; L^{2}(0, L)\right)}\|v-\| \|_{L^{2}\left(0, T ; L^{\infty}(0, L)\right)}\right\}
\end{aligned}
$$


Now, let us recall the classical interpolation inequality (Gagliardo-Niremberg)

$$
\|u\|_{L^{\infty}(0, L)} \leq C\|u\|_{L^{2}(0, L)}^{\frac{1}{2}}\left\|u_{x}\right\|_{L^{2}(0, L)}^{\frac{1}{2}}, \quad \forall u \in H_{0}^{1}(0, L) .
$$

As a consequence, we have

$$
\|u\|_{L^{2}\left(0, T ; L^{\infty}(0, L)\right)} \leq C T^{\frac{1}{4}}\|u\|_{L^{\infty}\left(0, T ; L^{2}(0, L)\right)}^{\frac{1}{2}}\|u\|_{L^{2}\left(0, T ; H_{0}^{1}(0, L)\right)}^{\frac{1}{2}} .
$$

Combining (20) and (21), we deduce that

$$
\|\Phi(v)-\Phi(w)\|_{X_{T}} \leq C(L+T) T^{\frac{1}{4}}\|u\|_{X_{T}}\|v-w\|_{X_{T}} .
$$

This shows that $\Phi$ is a contraction in the ball $B_{R}$ of $X_{T}$ if

$$
C(L+T) T^{\frac{1}{4}}\|u\|_{X_{T}}<1 .
$$

Therefore, the proof will be complete if we show that for a suitable choice of $R$ and $T$ satisfying (23), the map $\Phi$ sends $B_{R}$ into itself. Putting all the previous estimates together, we have

$$
\|\Phi(v)\|_{X_{T}} \leq C(L+T)\left\|v_{0}\right\|_{L^{2}(0, L)}+C(L+T) T^{\frac{1}{4}}\|u\|_{X_{T}}\|v\|_{X_{T}},
$$

or

$$
\|\Phi(v)\|_{X_{T}} \leq C(L+T)\left\|v_{0}\right\|_{L^{2}(0, L)}+C(L+T) T^{\frac{1}{4}}\|u\|_{X_{T}} R, \quad \forall v \in B_{R} .
$$

We then take $R=2 C(L+1)\left\|v_{0}\right\|_{L^{2}(0, L)}$. For this choice of $R$, the estimate above becomes

$$
\|\Phi(v)\|_{X_{T}} \leq\left\{C(L+T)+2 C^{2}(L+T)(L+1) T^{\frac{1}{4}}\|u\|_{X_{T}}\right\}\left\|v_{0}\right\|_{L^{2}(0, L)} .
$$

In order to guarantee that the right-hand side of $(25)$ is less than $R$, we need to choose $0<T<1$ sufficiently small such that

$$
2 C(L+T)(L+1) T^{\frac{1}{4}}\|u\|_{X_{T}} \leq C(L+1)
$$

which is always possible. Taking $T>0$ possibly smaller allows us to guarantee (23) as well.

This shows that system (8) has an unique mild solution for $0 \leq t<T$, with $T$ small. Thus, in order to conclude the proof of Lemma 3.1 it is sufficient to prove that this solution exists globally. To do that we need some a priori estimates, which will be obtained in several steps.

We first multiply the equation in (8) by $v$ and integrate by parts over $(0, L)$ to obtain

$$
\frac{1}{2} \frac{\mathrm{d}}{\mathrm{d} t} \int_{0}^{L} v^{2} \mathrm{~d} x+\frac{1}{2} v_{x}^{2}(0, t)+\int_{0}^{L} a(x) v^{2} \mathrm{~d} x=\int_{0}^{L} u v v_{x} \mathrm{~d} x .
$$

Now, integrating from 0 to $T$ and applying Cauchy-Schwarz and Hölder's inequalities in the right hand side of (26), it follows that

$$
\begin{aligned}
\int_{0}^{L} v^{2}(x, T) \mathrm{d} x & \leq \int_{0}^{L} v_{0}^{2} \mathrm{~d} x+2 \int_{0}^{T} \int_{0}^{L}\left|u v v_{x}\right| \mathrm{d} x \mathrm{~d} t \\
& \leq \int_{0}^{L} v_{0}^{2} \mathrm{~d} x+2\left(\int_{0}^{T}\|u v\|_{L^{2}(0, L)}^{2} \mathrm{~d} t\right)^{\frac{1}{2}}\left(\int_{0}^{T}\left\|v_{x}\right\|_{L^{2}(0, L)}^{2} \mathrm{~d} t\right)^{\frac{1}{2}} \\
& \leq \int_{0}^{L} v_{0}^{2} \mathrm{~d} x+\int_{0}^{T}\|u\|_{L^{\infty}(0, L)}^{2}\|v\|_{L^{2}(0, L)}^{2} \mathrm{~d} t+\int_{0}^{T}\left\|v_{x}\right\|_{L^{2}(0, L)}^{2} \mathrm{~d} t .
\end{aligned}
$$


In order to estimate the last term in the right hand side of (27), we multiply equation in (8) by $x v$ and integrate over $(0, L) \times(0, T)$. Then, performing integration by parts and using the boundary conditions we get

$$
\begin{aligned}
\int_{0}^{T} \int_{0}^{L} v_{x}^{2} \mathrm{~d} x \mathrm{~d} t+\frac{1}{3} \int_{0}^{L} x v^{2}(x, T) \mathrm{d} x+\frac{2}{3} \int_{0}^{T} \int_{0}^{L} x a(x) v^{2} \mathrm{~d} x \mathrm{~d} t \\
=\frac{1}{3} \int_{0}^{T} \int_{0}^{L} v^{2} \mathrm{~d} x \mathrm{~d} t+\frac{1}{3} \int_{0}^{L} x v_{0}^{2}(x) \mathrm{d} x+\frac{2}{3} \int_{0}^{T} \int_{0}^{L} x u v v_{x} \mathrm{~d} x \mathrm{~d} t+\frac{2}{3} \int_{0}^{T} \int_{0}^{L} u v^{2} \mathrm{~d} x \mathrm{~d} t
\end{aligned}
$$

or

$$
\int_{0}^{T} \int_{0}^{L} v_{x}^{2} \mathrm{~d} x \mathrm{~d} t \leq \frac{2}{3} \int_{0}^{T} \int_{0}^{L} x u v v_{x} \mathrm{~d} x \mathrm{~d} t+\frac{2}{3} \int_{0}^{T} \int_{0}^{L} u v^{2} \mathrm{~d} x \mathrm{~d} t+\frac{1}{3} \int_{0}^{T} \int_{0}^{L} v^{2} \mathrm{~d} x \mathrm{~d} t+\frac{1}{3} \int_{0}^{L} x v_{0}^{2}(x) \mathrm{d} x
$$

since the other terms that appears in the first line of (28) are positive. Thus, proceeding as in (27) and using Poincaré's inequality, we deduce that

$$
\int_{0}^{T} \int_{0}^{L} v_{x}^{2} \mathrm{~d} x \mathrm{~d} t \leq \frac{c}{\delta} \int_{0}^{T}\|u\|_{L^{\infty}(0, L)}^{2}\|v\|_{L^{2}(0, L)}^{2} \mathrm{~d} t+c \delta \int_{0}^{T} \int_{0}^{L} v_{x}^{2} \mathrm{~d} x \mathrm{~d} t+\frac{1}{3} \int_{0}^{T} \int_{0}^{L} v^{2} \mathrm{~d} x \mathrm{~d} t+\frac{L}{3} \int_{0}^{L} v_{0}^{2}(x) \mathrm{d} x
$$

where $c$ and $\delta$ are positive constants. Consequently, for sufficiently small $\delta$ we obtain

$$
\int_{0}^{T} \int_{0}^{L} v_{x}^{2} \mathrm{~d} x \mathrm{~d} t \leq C\left\{\int_{0}^{L} v_{0}^{2}(x) \mathrm{d} x+\int_{0}^{T}\left(1+\|u\|_{L^{\infty}(0, L)}^{2}\right)\|v\|_{L^{2}(0, L)}^{2} \mathrm{~d} t\right\},
$$

for some positive constant $C$. So, replacing (31) into (27) we can apply Gronwall's inequality and Theorem 2.1 to deduce that

$$
\|v\|_{L^{\infty}\left(0, T ; L^{2}(0, L)\right)} \leq C,
$$

where $C=C\left(T,\left\|u_{0}\right\|_{L^{2}(0, L)},\left\|v_{0}\right\|_{L^{2}(0, L)}\right)>0$. On the other hand, combining (31), (32) and Theorem 2.1, we obtain

$$
\|v\|_{L^{2}\left(0, T ; H_{0}^{1}(0, L)\right)} \leq C
$$

where $C>0$ also depends on $T,\left\|u_{0}\right\|_{L^{2}(0, L)}$ and $\left\|v_{0}\right\|_{L^{2}(0, L)}$. This concludes the proof of Lemma 3.1.

Lemma 3.2. There exists a positive constant $C=C\left(T,\left\|u_{0}\right\|_{L^{2}(0, L)}\right)$ such that

$$
\left\|v_{0}\right\|_{L^{2}(0, L)}^{2} \leq C\left\{\int_{0}^{T} v_{x}^{2}(0, t) \mathrm{d} t+\int_{0}^{T} \int_{0}^{L} a(x) v^{2} \mathrm{~d} x \mathrm{~d} t+\left\|v_{0}\right\|_{H^{-3}(0, L)}^{2}\right\},
$$

holds for every solution $v$ of (8) as in Lemma 3.1

Proof. To prove (34) we combine multiplier techniques and the so called "compactness-uniqueness" argument.

Multiplying equation in (8) by $(T-t) v$ and integrating over $(0, L) \times(0, T)$, we obtain

$$
\begin{aligned}
T\left\|v_{0}\right\|_{L^{2}(0, L)}^{2}= & \int_{0}^{T} \int_{0}^{L} v^{2} \mathrm{~d} x \mathrm{~d} t+\int_{0}^{T}(T-t) v_{x}^{2}(0, t) \mathrm{d} t+2 \int_{0}^{T} \int_{0}^{L}(T-t) a(x) v^{2} \mathrm{~d} x \mathrm{~d} t \\
& +\int_{0}^{T} \int_{0}^{L}(T-t) u_{x} v^{2} \mathrm{~d} x \mathrm{~d} t .
\end{aligned}
$$


From (35) we deduce that

$$
\left\|v_{0}\right\|_{L^{2}(0, L)}^{2} \leq \frac{1}{T} \int_{0}^{T} \int_{0}^{L} v^{2} \mathrm{~d} x \mathrm{~d} t+\int_{0}^{T} v_{x}^{2}(0, t) \mathrm{d} t+2 \int_{0}^{L} \int_{0}^{L} a(x) v^{2} \mathrm{~d} x \mathrm{~d} t+\int_{0}^{T} \int_{0}^{L}\left|u_{x}\right| v^{2} \mathrm{~d} x \mathrm{~d} t,
$$

and since

$$
\begin{aligned}
\int_{0}^{T} \int_{0}^{L}\left|u_{x}\right| v^{2} \mathrm{~d} x \mathrm{~d} t & \leq \int_{0}^{T}\left\|u_{x}\right\|_{L^{2}(0, L)}\|v\|_{L^{4}(0, L)}^{2} \mathrm{~d} t \\
& \leq\|u\|_{L^{2}\left(0, T ; H_{0}^{1}(0, L)\right)}\|v\|_{L^{4}\left(0, T ; L^{4}(0, L)\right)}^{2}
\end{aligned}
$$

it follows from (36) and Theorem 2.1 that

$$
\left\|v_{0}\right\|_{L^{2}(0, L)}^{2} \leq c\|v\|_{L^{4}\left(0, T ; L^{4}(0, L)\right)}^{2}+\int_{0}^{T} v_{x}^{2}(0, t) \mathrm{d} t+2 \int_{0}^{L} \int_{0}^{L} a(x) v^{2} \mathrm{~d} x \mathrm{~d} t,
$$

where $c>0$ only depends on $T$ and $\left\|u_{0}\right\|_{L^{2}(0, L)}$. Thus, in order to prove (34) it is sufficient to show that for any $T>0$ there exists a positive constant $C=C(T)$ such that

$$
\|v\|_{L^{4}\left(0, T ; L^{4}(0, L)\right)}^{2} \leq C\left\{\int_{0}^{T} v_{x}^{2}(0, t) \mathrm{d} t+\int_{0}^{L} \int_{0}^{L} a(x) v^{2} \mathrm{~d} x \mathrm{~d} t+\left\|v_{0}\right\|_{H^{-3}(0, L)}^{2}\right\}
$$

for any solution of (1).

We argue by contradiction. Suppose that (39) does not hold. Then, there exists a sequence of functions $v_{n} \in L^{\infty}\left(0, T ; L^{2}(0, L)\right) \cap L^{2}\left(0, T ; H_{0}^{1}(0, L)\right)$ that solves (1), satisfying

$$
\lim _{n \rightarrow \infty} \frac{\left\|v_{n}\right\|_{L^{4}\left(0, T ; L^{4}(0, L)\right)}^{2}}{\int_{0}^{T}\left|v_{n, x}(0, t)\right|^{2} \mathrm{~d} t+\int_{0}^{T} \int_{0}^{L} a(x) v_{n}^{2} \mathrm{~d} x \mathrm{~d} t+\left\|v_{0, n}\right\|_{H^{-3}(0, L)}^{2}}=\infty .
$$

Let $\lambda_{n}=\left\|v_{n}\right\|_{L^{4}\left(0, T ; L^{4}(0, L)\right)}$ and define $w_{n}(x, t)=\frac{v_{n}(x, t)}{\lambda_{n}}$. For each $n \in \mathbb{N}$ the function $w_{n}$ satisfies

$$
\left\{\begin{array}{l}
w_{n, t}+w_{n, x}+w_{n, x x x}+\left(u(x, t) w_{n}\right)_{x}+a(x) w_{n}=0 \quad \text { in }(0, L) \times(0, T), \\
w_{n}(0, t)=w_{n}(L, t)=w_{n, x}(L, t)=0, \quad t \in(0, T), \\
w_{n}(x, 0)=\frac{v_{n}(x, 0)}{\lambda_{n}}, \quad \text { on }(0, L) .
\end{array}\right.
$$

Moreover,

$$
\left\|w_{n}\right\|_{L^{4}\left(0, T ; L^{4}(0, L)\right)}=1
$$

and

$$
\int_{0}^{T}\left|w_{n, x}(0, t)\right|^{2} \mathrm{~d} t+\int_{0}^{L} \int_{0}^{L} a(x) w_{n}^{2} \mathrm{~d} x \mathrm{~d} t+\left\|w_{n}(., 0)\right\|_{H^{-3}(0, L)}^{2} \rightarrow 0
$$

as $n \rightarrow \infty$.

Using (38), (42) and (43) it follows that $w_{n}(x, 0)$ is bounded in $L^{2}(0, L)$, and therefore, according to (33),

$$
\left\|w_{n}\right\|_{L^{2}\left(0, T ; H_{0}^{1}(0, L)\right)} \leq C
$$


for some constant $C>0$. On the other hand,

$$
\left\|\left(u w_{n}\right)_{x}\right\|_{L^{2}\left(0, T ; L^{1}(0, L)\right)} \leq\left\|w_{n}\right\|_{L^{\infty}\left(0, T ; L^{2}(0, L)\right)}\|u\|_{L^{2}\left(0, T ; H_{0}^{1}(0, L)\right)}+\|u\|_{L^{\infty}\left(0, T ; L^{2}(0, L)\right)}\left\|w_{n}\right\|_{L^{2}\left(0, T ; H_{0}^{1}(0, L)\right)} .
$$

So, by (45) we obtain that there exists $C>0$ such that

$$
\left\|\left(u w_{n}\right)_{x}\right\|_{L^{2}\left(0, T ; L^{1}(0, L)\right)} \leq C
$$

and therefore,

$$
\left(w_{n}\right)_{t} \text { is bounded in } L^{2}\left(0, T ; H^{-2}(0, L)\right) \text {. }
$$

Indeed, according to $(41), w_{n, t}$ satisfies

$$
w_{n, t}=-w_{n, x}-w_{n, x x x}-\left(u(x, t) w_{n}\right)_{x}-a(x) w_{n} \quad \text { in } \mathcal{D}^{\prime}\left(0, T ; H^{-2}(0, L)\right),
$$

and (44)-(46) guarantee the boundedness (in $L^{2}\left(0, T ; H^{-2}(0, L)\right)$ ) of the terms appearing in the right hand side of the above equation.

Now we claim that the following holds: there exists $s>0$ such that $\left\{w_{n}\right\}$ is bounded in $L^{4}\left(0, T ; H^{s}(0, L)\right)$, the embedding $H^{s}(0, L) \hookrightarrow L^{4}(0, L)$ being compact.

In fact, since $\left\{w_{n}\right\}$ is bounded in $L^{2}\left(0, T ; H_{0}^{1}(0, L)\right) \cap L^{\infty}\left(0, T ; L^{2}(0, L)\right)$ by interpolation we can deduce that $\left\{w_{n}\right\}$ is bounded in

$$
\left[L^{q}\left(0, T ; L^{2}(0, L)\right), L^{2}\left(0, T ; H_{0}^{1}(0, L)\right)\right]_{\theta}=L^{p}\left(0, T ;\left[L^{2}(0, L), H_{0}^{1}(0, L)\right]_{\theta}\right)
$$

where $\frac{1}{p}=\frac{1-\theta}{q}+\frac{\theta}{2}$ and $0<\theta<1$. Thus, choosing $p=4, q=5$ and $\theta=1 / 6$, the claim holds with $s=5 / 6$, i.e.,

$$
\left[L^{2}(0, L), H_{0}^{1}(0, L)\right]_{\frac{1}{6}}=H^{\frac{5}{6}}(0, L) .
$$

Furthermore, the embedding $H^{\frac{5}{6}}(0, L) \hookrightarrow L^{4}(0, L)$ is compact.

Then, using the statement above, (47) and classical compactness results [18] (Cor. 4), we can extract a subsequence of $\left\{w_{n}\right\}$, that we also denote by $\left\{w_{n}\right\}$, such that

$$
w_{n} \rightarrow w \text { strongly in } L^{4}\left(0, T ; L^{4}(0, L)\right),
$$

and by (42),

$$
\|w\|_{L^{4}\left(0, T ; L^{4}(0, L)\right)}=1 .
$$

Moreover,

$$
\begin{aligned}
0 & =\liminf _{n \rightarrow \infty}\left\{\int_{0}^{T}\left|w_{n, x}\right|^{2} \mathrm{~d} t+\int_{0}^{L} \int_{0}^{L} a(x) w_{n}^{2} \mathrm{~d} x \mathrm{~d} t+\left\|w_{n}(., 0)\right\|_{H^{-3}(0, L)}^{2}\right\} \\
& \geq \int_{0}^{T}\left|w_{x}(0, t)\right|^{2} \mathrm{~d} t+\int_{0}^{L} \int_{0}^{L} a(x) w^{2} \mathrm{~d} x \mathrm{~d} t+\|w(., 0)\|_{H^{-3}(0, L)}^{2} .
\end{aligned}
$$


This implies, in particular, that $w(x, 0)=0$. Consequently, the limit $w$, which solves the system

$$
\left\{\begin{array}{l}
w_{t}+w_{x}+w_{x x x}+(u(x, t) w)_{x}+a(x) w=0 \quad \text { in }(0, L) \times(0, T), \\
w(0, t)=w(L, t)=0, \quad t \in(0, T), \\
w_{x}(L, t)=0, \quad t \in(0, T) \\
w(x, 0)=0, \quad \text { on }(0, L)
\end{array}\right.
$$

is identically zero, i.e., $w \equiv 0$. This contradicts (49) and, necessarily, (39) has to be valid. This completes the proof of Lemma 3.2 .

We are now in conditions to prove Theorem 2.2.

Proof of Theorem 2.2. Let $u_{0} \in L^{2}(0, L)$.

Differentiating equation in (1) with respect to $t$, we obtain system (8) with

$$
v_{0}(x)=v(x, 0)=u_{t}(x, 0)=-u_{0, x}-u_{0, x x x}-u_{0} u_{0, x}-a(x) u_{0} \in H^{-3}(0, L) .
$$

On the other hand, if $u_{x}(0, t)=0$ and $a(x) u$ vanishes then, $v_{x}(0, t)=0$ and $a(x) v \equiv 0$ as well. Consequently, by the assumption (2) on the damping potential $a=a(x), v \equiv 0$ in $\omega \times(0, T)$ and according to (2) and Lemma 3.2 we obtain that $v_{0} \in L^{2}(0, L)$. Now, combining Lemma 3.1 and system (1) we get

$$
u_{t}=v \in L^{\infty}\left(0, T ; L^{2}(0, L)\right) \cap L^{2}\left(0, T ; H_{0}^{1}(0, L)\right)
$$

and

$$
\left\{\begin{array}{l}
u_{x x x}=-u_{t}-u_{x}-u u_{x}-a(x) u, \quad \text { in }(0, L) \times(0, T), \\
u(0, t)=u(L, t)=0, \quad t \in(0, T) .
\end{array}\right.
$$

Thus, Theorem 2.1 together with (52) and (53) allow us to conclude that $u \in L^{2}\left(0, T ; H^{3}(0, L)\right) \cap$ $H^{1}\left(0, T ; L^{2}(0, L)\right)$. Finally, using the UCP proved in [17] (Cor. 1.2 and Th. 4.2) we have $u \equiv 0$.

\section{Further comments}

The methods of this paper allow to handle systems of dispersive equations, like the following one in [1]:

$$
\left\{\begin{array}{l}
u_{t}+u_{x}+u_{x x x}+a_{3} v_{x x x}+u u_{x}+a_{1} v v_{x}+a_{2}(u v)_{x}+a(x) u=0 \\
b_{1} v_{t}+v_{x}+v_{x x x}+b_{2} a_{3} u_{x x x}+v v_{x}+b_{2} a_{2} u u_{x}+b_{2} a_{1}(u v)_{x}+a(x) v=0,
\end{array}\right.
$$

with $0<x<L, t>0$, boundary conditions

and initial data

$$
\begin{gathered}
u(0, t)=u(L, t)=0, \quad v(0, t)=v(L, t)=0 \quad t \in(0, T), \\
u_{x}(L, t)=0, \quad v_{x}(L, t)=0, \quad t \in(0, T)
\end{gathered}
$$

$$
u(x, 0)=u_{0}(x), \quad v(x, 0)=v_{0}(x), \quad 0<x<L .
$$

In (54), $a_{1}, a_{2}, a_{3}, b_{1}$ and $b_{2}$ are assumed to be real constants with $b_{1}>0$ and $b_{2}>0$. When $a \equiv 0$ and $x$ runs in the whole line, this system of KdV equations was derived by Gear and Grimshaw [4] as a model describing strong interactions of weakly nonlinear long waves. 
The energy associated to the above model is given by

$$
E(t)=\frac{1}{2} \int_{0}^{L}\left(u^{2}+b_{1} v^{2}\right) \mathrm{d} x,
$$

and we can (formally) verify that

$$
\begin{aligned}
\frac{\mathrm{d} E}{\mathrm{~d} t}= & -\int_{0}^{L} a(x)\left(u^{2}+v^{2}\right) \mathrm{d} x-\frac{1}{2}\left[u_{x}^{2}(0, t)+v_{x}^{2}(0, t)+2 a_{3} u_{x}(0, t) v_{x}(0, t)\right] \\
& +\left(1-b_{2}\right) \int_{0}^{L}\left(a_{2} v u u_{x}-a_{3} u_{x x x} v-a_{1} u v v_{x}\right) \mathrm{d} x
\end{aligned}
$$

In [1] the analogue of the main result in [12] on the exponential decay of solutions was proved in the context of system (54). Namely, it was proved that the solutions tend exponentially to zero when the damping term $a=a(x)$ is effective on a neighborhood of both extremes of the boundary of the domain where equation holds. This was done under suitable conditions on the parameters entering in (54). The methods developed in this paper allow showing that the same exponential decay property holds when the damping potential $a=a(x)$ is effective in any non-empty subinterval.

\section{AN OPEN PROBLEM}

As we mentioned in the introduction the problem of the decay of solutions of (1) makes sense also in the absence of the damping potential $a$, i.e., when $a \equiv 0$. Indeed, according to the energy dissipation law (3) we have

$$
\frac{\mathrm{d} E}{\mathrm{~d} t}=-\frac{1}{2}\left|u_{x}(0, t)\right|^{2}
$$

In the linear case, as shown in [12,15] (see also [2]), there are critical lengths $L$ for which the decay is not true (the lengths $L$ is the set $\mathcal{E}$ in (9)). Whether solutions of the nonlinear problem decay in these cases or not is an open problem. Moreover, when $L \notin \mathcal{E}$, the decay of the linear semigroup allows showing that small amplitude solutions of the nonlinear problem decay exponentially as well. The decay of large solutions is also open.

Acknowledgements. The author would like to thank Professor Enrique Zuazua for helpful conversations they had while this research was in progress. This work was done when he was visiting Universidad Autónoma de Madrid in 2003 with the financial support of Grant SB2001-0158 of the MECD (Spain) and Grants BFM 2002-03345 of the MCYT (Spain).

\section{REFERENCES}

[1] E. Bisognin, V. Bisognin and G.P. Menzala, Exponential stabilization of a coupled system of Korteweg-de Vries Equations with localized damping. Adv. Diff. Eq. 8 (2003) 443-469.

[2] J. Coron and E. Crepéau, Exact boundary controllability of a nonlinear KdV equation with critical lengths. J. Eur. Math. Soc. 6 (2004) 367-398.

[3] B. Dehman, G. Lebeau and E. Zuazua, Stabilization and control for the subcritical semilinear wave equation. Ann. Sci. École Norm. Sup. 36 (2003) 525-551.

[4] J.A. Gear and R. Grimshaw, Weak and strong interaction between internal solitary waves. Stud. Appl. Math. 70 (1984) $235-258$.

[5] L. Hörmander, Linear partial differential operators. Springer Verlag, Berlin/New York (1976)

[6] L. Hörmander, The analysis of linear partial differential operators (III-IV). Springer-Verlag, Berlin (1985).

[7] O. Yu Imanuvilov and M. Yamamoto, Carleman estimate for a parabolic equation in Sobolev spaces of negative order and its applications, in Control of Nonlinear Distributed Parameter Systems, G. Chen et al. Eds. Marcel-Dekker (2001) 113-137. 
[8] T. Kato, On the Cauchy problem for the (generalized) Korteweg-de Vries equation. Stud. Appl. Math. Adv., in Math. Suppl. Stud. 8 (1983) 93-128.

[9] D.J. Korteweg and G. de Vries, On the change of form of long waves advancing in a retangular canal, and on a new type of long stacionary waves. Philos. Mag. 39 (1895) 422-423.

[10] S.N. Kruzhkov and A.V. Faminskii, Generalized solutions of the Cauchy problem for the Korteweg-de Vries equation. Math. URSS Sbornik 38 (1984) 391-421.

[11] J. Lions, Contrôlabilité exacte, perturbations et stabilization de systèmes distribué, Tome 1, Contrôlabilité exacte, Colletion de Recherches en Mathématiques Appliquées, Masson, Paris 8 (1988).

[12] G.P. Menzala, C.F. Vasconcellos and E. Zuazua, Stabilization of the Korteweg-de Vries equation with localized damping. Quarterly Appl. Math. LX (2002) 111-129.

[13] G.P. Menzala and E. Zuazua, Decay rates for the von Kàrmàn system of thermoelastic plates. Diff. Int. Eq. 11 (1998) 755-770.

[14] J. Rauch and M. Taylor, Exponential decay of solutions to symmetric hyperbolic equations in bounded domains. Indiana J. Math. 24 (1974) 79-86.

[15] L. Rosier, Exact boundary controllability for the Korteweg-de Vries equation on a bonded domain. ESAIM: COCV 2 (1997) $33-55$.

[16] A. Ruiz, Unique continuation for weak solutions of the wave equation plus a potential. J. Math. Pures Appl. 71 (1992) $455-467$.

[17] J.C. Saut and B. Scheurer, Unique Continuation for some evolution equations. J. Diff. Equations 66 (1987) 118-139.

[18] J. Simon, Compact sets in the space $L^{p}(0, T ; B)$. Annali di Matematica Pura ed Appicata CXLVI (IV) (1987) 65-96.

[19] F. Trêves, Linear Partial Differential Equations. Gordon and Breach, New York/London/Paris (1970).

[20] B.Y. Zhang, Unique continuation for the Korteweg-de Vries equation. SIAM J. Math. Anal. 23 (1992) 55-71.

[21] B.Y. Zhang, Exact boundary controllability of the Kortewed-de Vries equation. SIAM J. Control Opt. 37 (1999) 543-565.

[22] E. Zuazua, Contrôlabilité exacte de quelques modèles de plaques en un temps arbitrairement petit, Appendix I in [11] 465-491.

[23] E. Zuazua, Exponential decay for the semilinear wave equation with locally distributed damping. Comm. Partial Diff. Eq. 15 (1990) 205-235.

[24] C. Zuily, Uniqueness and nonuniqueness in the Cauchy problem. Birkhäuser, Progr. Math. 33 (1983). 